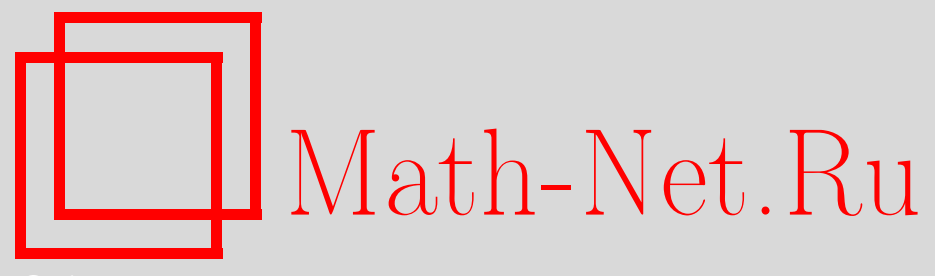

А. А. Андреев, Ю. О. Яковлева, Задача Коши для уравнения гиперболического типа порядка $n$ общего вида с некратными характеристиками, Вестн. Сам. гос. техн. ун-та. Сер. Физ.-мат. науки, 2016, номер 2, 241-248

DOI: https://doi.org/10.14498/vsgtu1490

Использование Общероссийского математического портала MathNet.Ru подразумевает, что вы прочитали и согласны с пользовательским соглашением

http://www.mathnet.ru/rus/agreement

Параметры загрузки:

IP : 34.239 .49 .27

26 апреля 2023 г., 05:17:25

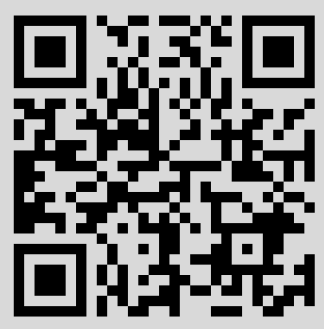


УДК 517.956.3

\title{
ЗАДАЧА КОШИ ДЛЯ УРАВНЕНИЯ ГИПЕРБОЛИЧЕСКОГО ТИПА ПОРЯДКА $N$ ОБЩЕГО ВИДА С НЕКРАТНЫМИ ХАРАКТЕРИСТИКАМИ
}

\author{
А. А. Андреев, Ю.О. Яковлева \\ 1 Самарский государственный технический университет, \\ Россия, 443100, Самара, ул. Молодогвардейская, 244. \\ 2 Самарский национальный исследовательский университет \\ имени академика С.П. Королева, \\ Россия, 443086, Самара, Московское ш., 34
}

\section{Аннотация}

Для дифференциального уравнения гиперболического типа порядка $n$ с некратными характеристиками рассмотрена задача Коши. Приводятся полученные авторами ранее решения задачи Коши для гиперболических уравнений третьего и четвертого порядков с некратными характеристиками в явном виде, аналогичном формуле Даламбера. Получено решение задачи Коши для уравнения гиперболического типа порядка $n$ общего вида. Найденное решение также является аналогом формулы Даламбера. Сформулирована теорема о существовании и единственности регулярного решения задачи Коши для гиперболического уравнения порядка $n$ общего вида с некратными характеристиками.

Ключевые слова: дифференциальное уравнение гиперболического типа порядка $n$, некратные характеристики, метод общих решений, задача Коши, формула Даламбера.

Введение. Метод общих решений в применении к решению задачи Коши для дифференциальных уравнений гиперболического типа имеет большую историю. Известен классический результат решения задачи Коши для гиперболического уравнения второго порядка, получивший название формулы Даламбера [1-4]. В дальнейшем в своих исследованиях к этой теме обращались как отечественные, так и зарубежные ученые [5-9]. Некоторая часть работ авторов данной статьи также посвящена этой теме.

1. Предварительные сведения. В работе [10] приведено решение задачи Коши для гиперболического уравнения третьего порядка

$$
a_{0} u_{x x x}+a_{1} u_{x x y}+a_{2} u_{x y y}+a_{3} u_{y y y}=0,
$$

(C) 2016 Самарский государственный технический университет.

\section{Образец для цитирования}

Андреев А.А., Яковлева Ю. О. Задача Коши для уравнения гиперболического типа порядка $n$ общего вида с некратными характеристиками // Вестн. Сам. гос. техн. ун-та. Сер. Физ.-мат. науки, 2016. Т. 20, № 2. С. 241-248. doi: 10.14498/vsgtu1490.

\section{Сведения об авторах}

Александр Анатольевич Андреев (к.ф.-м.н., доц.; andre01071948@yandex.ru), доцент, каф. прикладной математики и информатики.

Юлия Олеговна Яковлева (к.ф.-м.н.; julia.yakovleva@mail .ru; aвтор, ведущий переписку), доцент, каф. математики и бизнес-информатики. 
где $a_{0}, a_{1}, a_{2}, a_{3}$ - некоторые действительные положительные постоянные. Корни характеристического уравнения для (1) $\lambda_{1}, \lambda_{2}, \lambda_{3}$ отличны от нуля и такие, что ${ }^{1}$

$$
\lambda_{1}+\lambda_{2}+\lambda_{3}=-\frac{a_{1}}{a_{0}}, \quad \lambda_{1} \lambda_{2}+\lambda_{2} \lambda_{3}+\lambda_{1} \lambda_{3}=\frac{a_{2}}{a_{0}}, \quad \lambda_{1} \lambda_{2} \lambda_{3}=-\frac{a_{3}}{a_{0}} .
$$

Решением задачи Коши для уравнения (1) с условиями

$$
\left.u(x, y)\right|_{y=0}=\alpha(x),\left.\quad \frac{\partial u}{\partial n}\right|_{y=0}=\beta(x),\left.\quad \frac{\partial^{2} u}{\partial n^{2}}\right|_{y=0}=\gamma(x), \quad x \in \mathbb{R},
$$

где $\alpha(x), \beta(x), \gamma(x) \in C^{3}(\mathbb{R}), n=\{0,1\}$ - нормаль к нехарактеристической прямой $y=0$, является функция

$$
u(x, y)=\sum_{k=1}^{3} \frac{\lambda_{k}^{3}}{\lambda_{k}^{3}-\lambda_{k}^{2}\left(\frac{a_{1}}{a_{0}}-\lambda_{k}\right)+\frac{a_{3}}{a_{0}}} F\left(x, y, \lambda_{k}\right) \in C^{3}\left(\mathbb{R}^{2}\right),
$$

где

$$
\begin{gathered}
F(x, y, \lambda)=\alpha\left(x-\frac{y}{\lambda}\right)+\frac{a_{1}-\lambda a_{0}}{a_{0}} \int_{0}^{x-\frac{y}{\lambda}} \beta(t) d t+ \\
+\frac{a_{3}}{a_{0} \lambda} \int_{0}^{x-\frac{y}{\lambda}} \gamma(t)\left(x-\frac{y}{\lambda}-t\right) d t .
\end{gathered}
$$

Формула (2) в [10] была названа аналогом формулы Даламбера для гиперболического уравнения третьего порядка.

В работе [11] рассмотрено дифференциальное уравнение гиперболического типа четвертого порядка в частных производных общего вида

$$
a_{0} u_{x x x x}+a_{1} u_{x x x y}+a_{2} u_{x x y y}+a_{3} u_{x y y y}+a_{4} u_{y y y y}=0
$$

где $a_{0}, a_{1}, a_{2}, a_{3}, a_{4}$ - действительные ненулевые постоянные. Характеристическое уравнение для уравнения (3)

$$
a_{0} \lambda^{4}-a_{1} \lambda^{3}+a_{2} \lambda-a_{3} \lambda+a_{4}=0
$$

имеет четыре различных действительных отличных от нуля корня $\lambda_{1}, \lambda_{2}, \lambda_{3}$, $\lambda_{4}$ таких, что

$$
\begin{gathered}
\lambda_{1}+\lambda_{2}+\lambda_{3}+\lambda_{4}=-\frac{a_{1}}{a_{0}}, \quad \lambda_{1} \lambda_{2}+\lambda_{1} \lambda_{3}+\lambda_{1} \lambda_{4}+\lambda_{2} \lambda_{3}+\lambda_{2} \lambda_{4}+\lambda_{3} \lambda_{4}=\frac{a_{2}}{a_{0}}, \\
\lambda_{1} \lambda_{2} \lambda_{3}+\lambda_{1} \lambda_{2} \lambda_{4}+\lambda_{1} \lambda_{3} \lambda_{4}+\lambda_{2} \lambda_{3} \lambda_{4}=-\frac{a_{3}}{a_{0}}, \quad \lambda_{1} \lambda_{2} \lambda_{3} \lambda_{4}=\frac{a_{4}}{a_{0}} .
\end{gathered}
$$

В [11] поставлена и решена следующая задача Коши.

\footnotetext{
${ }^{1}$ В работах $[10,11]$ имеется опечатка, касающаяся свойств корней характеристического уравнения для (1), которая не влияет на полученные результаты.
} 
ЗАДАчА Коши. В плоскости $\mathbb{R}^{2}$ найти регулярное решение $u(x, y) \in C^{4}\left(\mathbb{R}^{2}\right)$ уравнения (3), удовлетворяющее условиям

$$
\left.u(x, y)\right|_{y=0}=\alpha(x),\left.\quad \frac{\partial u}{\partial n}\right|_{y=0}=\beta(x),\left.\quad \frac{\partial^{2} u}{\partial n^{2}}\right|_{y=0}=\gamma(x),\left.\quad \frac{\partial^{3} u}{\partial n^{3}}\right|_{y=0}=\sigma(x),
$$

где $\alpha(x), \beta(x), \gamma(x), \sigma(x) \in C^{4}(\mathbb{R}) ; n=\{0,1\}$ - нормаль к нехарактеристической линии $y=0$.

Регулярным решением задачи (3), (4) является функция

$$
u(x, y)=\sum_{k=1}^{4} \frac{(-1)^{k+1} \lambda_{k}^{3}}{\prod_{m=1, m \neq k}^{4}\left(\lambda_{k}-\lambda_{m}\right)} F\left(x, y, \lambda_{k}\right),
$$

где

$$
\begin{aligned}
F(x, y, \lambda)=\alpha(x & \left.-\frac{1}{\lambda} y\right)-\frac{a_{1}+a_{0} \lambda_{k}}{a_{0}} \int_{0}^{x-\frac{y}{\lambda_{k}}} \beta(t) d t- \\
& -\frac{a_{4}+a_{3} \lambda_{k}}{a_{0} \lambda_{k}^{2}} \int_{0}^{x-\frac{y}{\lambda_{k}}} \gamma(t)\left(x-\frac{y}{\lambda_{k}}-t\right) d t+ \\
& +\frac{a_{4}}{2 a_{0} \lambda_{k}} \int_{0}^{x-\frac{y}{\lambda_{k}}} \sigma(t)\left(x-\frac{y}{\lambda_{k}}-t\right)^{2} d t .
\end{aligned}
$$

Решение (5) задачи (3), (4) получено в виде, аналогичном формуле Даламбеpa.

Приведенные исследования можно обобщить на случай уравнения гиперболического типа порядка $n$.

2. Задача Коши для уравнения гиперболического типа порядка $\boldsymbol{n}$ общего вида с некратными характеристиками. Рассмотрим уравнения гиперболического типа порядка $n$

$$
a_{0} u_{x x x \ldots x}+a_{1} u_{x x \ldots x y}+\ldots+a_{n-1} u_{x \ldots y y y}+a_{n} u_{y y y \ldots y}=0,
$$

где $a_{0}, a_{1}, \ldots, a_{n-1}, a_{n}$ - действительные ненулевые постоянные.

Характеристическое уравнение для уравнения (6)

$$
a_{0} \lambda^{n}+a_{1} \lambda^{n-1}+\ldots+a_{n-1} \lambda+a_{n}=0
$$

имеет $n$ различных действительных отличных от нулей корня $\lambda_{1}, \lambda_{2}, \ldots, \lambda_{n}$ таких, что

$$
\begin{gathered}
\lambda_{1}+\lambda_{2}+\ldots+\lambda_{n}=-\frac{a_{1}}{a_{0}}, \quad \lambda_{1} \lambda_{2}+\lambda_{1} \lambda_{3}+\cdots+\lambda_{n-1} \lambda_{n}=\frac{a_{2}}{a_{0}}, \\
\lambda_{1} \lambda_{2} \lambda_{3}+\lambda_{1} \lambda_{2} \lambda_{4}+\ldots+\lambda_{n-2} \lambda_{n-1} \lambda_{n}=-\frac{a_{3}}{a_{0}}, \quad \ldots \\
\lambda_{1} \lambda_{2} \cdots \lambda_{n-1}+\lambda_{2} \lambda_{3} \cdots \lambda_{n}+\cdots+\lambda_{1} \lambda_{3} \cdots \lambda_{n}=(-1)^{n-1} \frac{a_{n-1}}{a_{0}} \\
\lambda_{1} \lambda_{2} \cdots \lambda_{n}=(-1)^{n} \frac{a_{n}}{a_{0}}
\end{gathered}
$$


Тогда уравнение (6) является строго гиперболическим по Петровскому [12].

Общее решение уравнения (6) из класса $n$ раз непрерывно дифференцируемых функций $C^{n}\left(\mathbb{R}^{2}\right)$ будет представляться в виде $[7,11]$

$$
u(x, y)=f_{1}\left(y+\lambda_{1} x\right)+f_{2}\left(y+\lambda_{2} x\right)+\ldots+f_{n}\left(y+\lambda_{n} x\right) .
$$

ЗАДАчА Коши. В плоскости $\mathbb{R}^{2}$ найти регулярное решение $u(x, y) \in C^{n}\left(\mathbb{R}^{2}\right)$ уравнения (6), удовлетворяющее условиям

$$
\begin{gathered}
\left.u(x, y)\right|_{y=0}=\tau_{n}(x),\left.\quad \frac{\partial u}{\partial n}\right|_{y=0}=\tau_{n-1}(x),\left.\quad \frac{\partial^{2} u}{\partial n^{2}}\right|_{y=0}=\tau_{n-2}(x), \ldots, \\
\left.\frac{\partial^{n-1} u}{\partial n^{n-1}}\right|_{y=0}=\tau_{1}(x),
\end{gathered}
$$

где $\tau_{n}(x), \tau_{n-1}(x), \tau_{n-2}(x), \ldots, \tau_{1}(x) \in C^{n}(\mathbb{R}), n=\{0,1\}$ - нормаль к нехарактеристической линии $y=0$.

Регулярным в плоскости $\mathbb{R}^{2}$ решением задачи Коши (6), (7) будем называть функцию $u(x, y) \in C^{n}\left(\mathbb{R}^{2}\right)$, имеющую в плоскости $\mathbb{R}^{2}$ все непрерывные частные производные, входящие в уравнение (6), и удовлетворяющую ему и условиям Коши (7) в обычном смысле.

Ограничения на нехарактеристическую линию уравнения порядка $n$ такие же, как и для уравнения второго порядка. Эта линия не может дважды пересекать любую характеристику из любого другого семейства [3,4].

Определим функции $f_{1}, f_{2}, \ldots, f_{n}$ так, чтобы удовлетворялись условия задачи Коши (7):

$$
\begin{gathered}
f_{1}\left(\lambda_{1} x\right)+f_{2}\left(\lambda_{2} x\right)+f_{3}\left(\lambda_{3} x\right)+\ldots+f_{n}\left(\lambda_{n} x\right)=\tau_{n}(x), \\
f_{1}^{\prime}\left(\lambda_{1} x\right)+f_{2}^{\prime}\left(\lambda_{2} x\right)+f_{3}^{\prime}\left(\lambda_{3} x\right)+\ldots+f_{n}^{\prime}\left(\lambda_{n} x\right)=\tau_{n-1}(x), \\
\ldots, \\
f_{1}^{(n-1)}\left(\lambda_{1} x\right)+f_{2}^{(n-1)}\left(\lambda_{2} x\right)+f_{3}^{(n-1)}\left(\lambda_{3} x\right)+\ldots+f_{n}^{(n-1)}\left(\lambda_{n} x\right)=\tau_{1}(x) .
\end{gathered}
$$

Продифференцировав каждое из указанных равенств, получим

$$
\begin{gathered}
f_{1}^{(n-1)}\left(\lambda_{1} x\right)+\ldots+f_{k}^{(n-1)}\left(\lambda_{k} x\right)+\ldots+f_{n}^{(n-1)}\left(\lambda_{n} x\right)=\tau_{1}(x), \\
\ldots, \\
\lambda_{1}^{n-2} f_{1}^{(n-1)}\left(\lambda_{1} x\right)+\ldots+\lambda_{k}^{n-2} f_{k}^{(n-1)}\left(\lambda_{k} x\right)+\ldots+\lambda_{n}^{n-2} f_{n}^{(n-1)}\left(\lambda_{n} x\right)=\tau_{n-1}^{(n-2)}(x), \\
\lambda_{1}^{n-1} f_{1}^{(n-1)}\left(\lambda_{1} x\right)+\ldots+\lambda_{k}^{n-1} f_{k}^{(n-1)}\left(\lambda_{k} x\right)+\ldots+\lambda_{n}^{n-1} f_{n}^{(n-1)}\left(\lambda_{n} x\right)=\tau_{n}^{(n-1)}(x),
\end{gathered}
$$

или в компактном виде

$$
\sum_{k=1}^{n} \lambda_{k}^{(j-1)} f_{k}^{n-1}\left(\lambda_{k} x\right)=\tau_{j}^{(j-1)}(x), \quad j=\overline{1, n} .
$$

Определитель системы (8) является определителем Вандермонда [13]

$$
\Delta=\prod_{1 \leqslant i<j \leqslant n}\left(\lambda_{j}-\lambda_{i}\right)
$$


При этом

$$
\begin{aligned}
\Delta_{k}=\prod_{1 \leqslant i<j \leqslant n}\left(\lambda_{j}-\lambda_{i}\right)\left[( - 1 ) ^ { n + k + 1 } \left(\frac{a_{n}}{a_{0}} \tau_{1}(x)\right.\right. & +\frac{a_{n-1}}{a_{0}} \tau_{2}^{\prime}(x)+\ldots \\
& \left.\left.+\frac{a_{s}}{a_{0}} \tau_{n-s+1}^{(n-s)}(x)+\tau_{n}^{(n-1)}(x)\right)\right] .
\end{aligned}
$$

Разрешая систему (8) относительно неизвестных функций, получим

$$
\begin{aligned}
f_{k}^{(n-1)}\left(\lambda_{k} x\right)=(-1)^{n+k+1} & \frac{a_{n}}{a_{0}} \tau_{1}(x)+(-1)^{n+k+1} \frac{a_{n-1}}{a_{0}} \tau_{2}^{\prime}(x)+\ldots \\
& +(-1)^{n+k+1} \frac{a_{s}}{a_{0}} \tau_{n-s+1}^{(n-s)}(x)+\ldots+(-1)^{n+k+1} \tau_{n}^{(n-1)}(x) .
\end{aligned}
$$

Проинтегрировав $n-1$ раз левую часть равенства (9), получим

$$
\int_{0}^{x} \cdots \int_{0}^{x} f_{k}^{(n-1)}\left(\lambda_{k} s\right) d s=\frac{1}{\lambda^{n-1}}\left[f_{k}(x)-\sum_{r=0}^{n-2} f^{(r)}(0) x^{r}\right] .
$$

Результатом $n-1$ интегрирования правой части равенства (9) является следующее выражение:

$$
\begin{aligned}
& \frac{(-1)^{n+k+1}}{(n-2) !} \frac{a_{n}}{a_{0}} \int_{0}^{x} \tau_{1}(s)(x-s)^{n-2} d s+ \\
& +\frac{(-1)^{n+k+1}}{(n-3) !} \frac{a_{n-1}}{a_{0}}\left[\int_{0}^{x} \tau_{2}(s)(x-s)^{n-3} d s-\tau_{2}(0) \frac{x^{n-2}}{(n-2) !}\right]+\ldots+ \\
& \quad+(-1)^{n+k+1}\left[\tau_{n}(x)-\tau_{n}^{(n-2)}(0) \frac{x^{n-2}}{(n-2) !}\right] .
\end{aligned}
$$

Из (10), (11) с учетом условий согласования получим

$$
\begin{aligned}
f_{k}\left(\lambda_{k} x\right)=\lambda_{k}^{n-1} & \frac{(-1)^{n+k+1}}{(n-2) !} \frac{a_{n}}{a_{0}} \int_{0}^{x} \tau_{1}(s)(x-s)^{n-2} d s+ \\
& +\lambda_{k}^{n-1} \frac{(-1)^{n+k+1}}{(n-3) !} \frac{a_{n-1}}{a_{0}} \int_{0}^{x} \tau_{2}(s)(x-s)^{n-3} d s+\ldots+ \\
& +\lambda_{k}^{n-1}(-1)^{n+k+1} \tau_{n}(x) .
\end{aligned}
$$

Подставляя в формулу общего решения найденные функции $f_{k}(12)$, получим

$$
u(x, y)=\sum_{k=1}^{n} f_{k}\left(y+\lambda_{k} x\right)
$$

Непосредственной подстановкой можно убедиться, что формула (13) удовлетворяет уравнению (6) и условиям Коши (7).

Формулу (13) будем называть аналогом формулы Даламбера для гиперболического уравнения порядка $n$. 
Приведенные исследования позволяют сформулировать следующую теорему.

Tеорема. Если $\tau_{1}(x), \tau_{2}(x), \ldots, \tau_{n}(x) \in C^{n}(\mathbb{R})$, mо существует единственное регулярное решение $u(x, y) \in C^{n}\left(\mathbb{R}^{2}\right)$ задачи Коши (6), (7), которое имеет вид (13).

\section{ORCID}

Александр Анатольевич Андреев: http://orcid.org/0000-0002-6611-6685

Юлия Олеговна Яковлева: http://orcid.org/0000-0002-9839-3740

\section{БИБЛИОГРАФИЧЕСКИЙ СПИСОК}

1. Holmgren E. Sur les systèmes linéaires aux dérivées partielles du premier ordre deux variables indépendantes à caractéristiques réelles et distinetes // Arkiv f. Mat., Astr. och Fys., 1909. vol. 5, no. 1. 13 pp. (In Swedish)

2. Rieman B. Ueber die Fortpflanzung ebener Luftwellen von endlicher Schwingungsweite (Aus dem achten Bande der Abhandlungen der Königlichen Gesellschaft der Wissenschaften zu Göttingen. 1860.) / Bernard Riemann's Gesammelte mathematische Werke und wissenschaftlicher Nachlass; eds. R. Dedekind, H. M. Weber. United States: BiblioLife, 2009. pp. 145-164 (In German). doi : 10.1017/cbo9781139568050.009.

3. Бицадзе А. В. Уравнения математической физики. М.: Наука, 1982. 336 с.

4. Тихонов А. Н., Самарский А. А. Уравнения математической физики. М.: Наука, 1972. $736 \mathrm{c.}$

5. Ali Raeisian S. M. Effective Solution of Riemann Problem for Fifth Order Improperly Elliptic Equation on a Rectangle// AJCM, 2012. vol. 2, no.4. pp. 282-286. doi : 10.4236/ajcm. 2012.24038.

6. Nikolov A., Popivanov N. Singular solutions to Protter's problem for (3+1)-D degenerate wave equation (8-13 June 2012; Sozopol, Bulgaria) / AIP Conf. Proc., 1497, 2012. pp. 233238. doi: 10.1063/1.4766790.

7. Корзюк В. И., Чеб Е. С., Ле Тхи Тху, Решение смешанной задачи для биволнового уравнения методом характеристик // Тр. Ин-та матем., 2010. Т. 18, № 2. С. 36-54.

8. Миронов А. Н. О методе Римана решения задачи Коши // Изв. вузов. Матем., 2005. № 2. C. 34-44.

9. Радкевич Е. В. О корректности задачи Коши и смешанной задачи для некоторого класса гиперболических систем и уравнений с постоянными коэффициентами и переменной кратностью характеристик / Трудь Четвертой Международной конференции по дифференциальным и функиионально-дифференииальным уравнениям (Москва, 14-21 августа, 2005). Часть 2/ СМФН, Т. 16. М.: РУДН, 2006. С. 110-135.

10. Яковлева Ю. О. Задача Коши для гиперболического уравнения и системы гиперболических уравнений третьего порядка с некратными характеристиками // Научные ведомости БелГУ. Сер. Математика. Физика, 2013. Т. 31, №11. С. 109-117.

11. Андреев А. А., Яковлева Ю. О. Задача Коши для системы уравнений гиперболического типа четвертого порядка общего вида с некратными характеристиками // Becmн. Сам. гос. техн. ун-та. Сер. Физ.-мат. науки, 2014. №4(37). С. 7-15. doi: 10.14498/ vsgtu1349.

12. Петровский И. Г. Избранные труды. Системы уравнений с частными производными. Алгебраическая геометрия. М.: Наука, 1986. 500 с.

13. Bellman R. Introduction to matrix analysis: 2nd ed., Reprint of the 1970 Orig. / Classics in Applied Mathematics. vol. 19. Philadelphia, PA: Society for Industrial and Applied Mathematics, 1997. xxviii+403 pp.

Поступила в редакцию 10/IV/2016;

в окончательном варианте $-21 / \mathrm{V} / 2016$;

принята в печать $-27 / \mathrm{V} / 2016$. 
Vestn. Samar. Gos. Techn. Un-ta. Ser. Fiz.-mat. nauki

[J. Samara State Tech. Univ., Ser. Phys. \& Math. Sci.], 2016, vol. 20, no. 2, pp. 241-248

ISSN: 2310-7081 (online), 1991-8615 (print)

doi: http://dx.doi.org/10.14498/vsgtu1490

MSC: 35L25

\title{
THE CAUCHY PROBLEM FOR A GENERAL HYPERBOLIC DIFFERENTIAL EQUATION OF THE N-TH ORDER WITH THE NONMULTIPLE CHARACTERISTICS
}

\author{
A. A. Andreev, J. O. Yakovleva \\ 1 Samara State Technical University, \\ 244, Molodogvardeyskaya st., Samara, 443100, Russian Federation. \\ 2 Samara National Research University, \\ 34, Moskovskoye shosse, Samara, 443086, Russian Federation.
}

\begin{abstract}
In the paper the problem of Cauchy is considered for the hyperbolic differential equation of the $n$-th order with the nonmultiple characteristics. The Cauchy problem is considered for the hyperbolic differential equation of the third order with the nonmultiple characteristics for example. The analogue of D'Alembert formula is obtained as a solution of the Cauchy problem for the hyperbolic differential equation of the third order with the nonmultiple characteristics. The regular solution of the Cauchy problem for the hyperbolic differential equation of the forth order with the nonmultiple characteristics is constructed in an explicit form. The regular solution of the Cauchy problem for the $n$-th order hyperbolic differential equation with the nonmultiple characteristics is constructed in an explicit form. The analogue of D'Alembert formula is obtained as a solution of this problem also. The existence and uniqueness theorem for the regular solution of the Cauchy problem for the $n$-th order hyperbolic differential equation with the nonmultiple characteristics is formulated as the result of the research.
\end{abstract}

Keywords: $n$-th order hyperbolic differential equation, nonmultiple characteristics, Cauchy problem, D'Alembert formula.

\section{ORCID}

Aleksandr A. Andreev: http://orcid.org/0000-0002-6611-6685

Julia O. Yakovleva: http://orcid.org/0000-0002-9839-3740

(C) 2016 Samara State Technical University.

Please cite this article in press as:

Andreev A. A., Yakovleva J. O. The Cauchy problem for a general hyperbolic differential equation of the n-th order with the nonmultiple characteristics, Vestn. Samar. Gos. Tekhn. Univ., Ser. Fiz.-Mat. Nauki [J. Samara State Tech. Univ., Ser. Phys. \& Math. Sci.], 2016, vol. 20, no. 2, pp. 241-248. doi: 10.14498/vsgtu1490. (In Russian)

\section{Authors Details:}

Aleksandr A. Andreev (Cand. Phys. \& Math. Sci.; andre01071948@yandex.ru), Associate Professor, Dept. of Applied Mathematics \& Computer Science.

Julia O. Yakovleva (Cand. Phys. \& Math. Sci.; julia.yakovleva@mail.ru; Corresponding Author), Associate Professor, Dept. of Mathematics \& Business Informatics. 


\section{REFERENCES}

1. Holmgren E. Sur les systèmes linéaires aux dérivées partielles du premier ordre deux variables indépendantes à caractéristiques réelles et distinetes, Arkiv f. Mat., Astr. och Fys., 1909, vol. 5, no. 1, 13 pp. (In Swedish)

2. Rieman B. Ueber die Fortpflanzung ebener Luftwellen von endlicher Schwingungsweite (Aus dem achten Bande der Abhandlungen der Königlichen Gesellschaft der Wissenschaften zu Göttingen. 1860.), Bernard Riemann's Gesammelte mathematische Werke und wissenschaftlicher Nachlass; eds. R. Dedekind, H. M. Weber. United States, BiblioLife, 2009, pp. 145-164 (In German). doi : 10.1017/cbo9781139568050.009.

3. Bitsadze A. V. Uravneniia matematicheskoi fiziki [Equations of mathematical physics]. Moscow, Nauka, 1982, 336 pp. (In Russian)

4. Tikhonov A. N., Samarskii A. A. Uravneniia matematicheskoi fiziki [Equations of Mathematical Physics]. Moscow, Nauka, 1972, 736 pp. (In Russian)

5. Ali Raeisian S. M. Effective Solution of Riemann Problem for Fifth Order Improperly Elliptic Equation on a Rectangle, AJCM, 2012, vol. 2, no. 4, pp. 282-286. doi : 10.4236/ajcm . 2012.24038.

6. Nikolov A., Popivanov N. Singular solutions to Protter's problem for (3+1)-D degenerate wave equation (8-13 June 2012; Sozopol, Bulgaria), AIP Conf. Proc., 1497, 2012, pp. 233238. doi : 10.1063/1.4766790.

7. Korzyuk V. I., Cheb E. S., Le Thi Thu Solution of the mixed problem for the biwave equation by the method of characteristics, Tr. Inst. Mat., 2010, vol. 18, no. 2, pp. 36-54 (In Russian).

8. Mironov A. N. On the Riemann method for solving the Cauchy problem, Russian Math. (Iz. VUZ), 2005, vol. 49, no. 2, pp. 32-41.

9. Radkevich E. V. On the well-posedness of the Cauchy problem and a mixed problem for a class of hyperbolic systems and equations with constant coefficients and variable multiplicity of characteristics, Journal of Mathematical Sciences, 2008, vol. 149, no. 5, pp. 1580-1607. doi: 10.1007/s10958-008-0083-3.

10. Yakovleva J. O. The Cauchy problem for the hyperbolic equation and hyperbolic equations system of the third order with nonmultiple characteristics, Nauchnye vedomosti BelGU. Ser. Matematika. Fizika, 2013, vol. 31, no. 11, pp. 109-117 (In Russian).

11. Andreev A. A., Yakovleva J. O. The Cauchy problem for the system of general hyperbolic differential equations of the forth order with nonmultiple characteristics, Vestn. Samar. Gos. Tekhn. Univ. Ser. Fiz.-Mat. Nauki [J. Samara State Tech. Univ., Ser. Phys. \& Math. Sci.], 2014, no. 4(37), pp. 7-15 (In Russian). doi: 10.14498/vsgtu1349.

12. Petrovsky I. G. Izbrannye trudy. Sistemy uravnenii s chastnymi proizvodnymi. Algebraicheskaia geometriia [Selected works. Systems of partial differential equations. Algebraic geometry]. Moscow, Nauka, 1986, 504 pp.

13. Bellman R. Introduction to matrix analysis, 2nd ed., Reprint of the 1970 Orig., Classics in Applied Mathematics, vol. 19. Philadelphia, PA, Society for Industrial and Applied Mathematics, 1997, xxviii+403 pp.

Received 10/IV/2016; received in revised form $21 / \mathrm{V} / 2016$; accepted 27/V/2016. 